\title{
Optimal timing of coronary artery bypass after acute myocardial infarction: A review of California discharge data
}

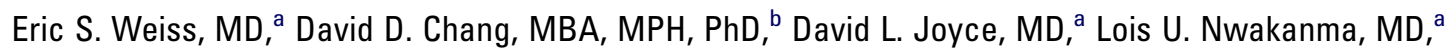
and David D. Yuh, MD

$\mathcal{B}$ Supplemental material is available online.
From the Division of Cardiac Surgery, ${ }^{\mathrm{a}} \mathrm{De}-$ partment of Surgery, ${ }^{b}$ The Johns Hopkins Medical Institutions, Baltimore, Maryland.

Presented at the 33rd Annual Meeting of the Western Thoracic Association, June 27 to 30, Santa Anna Pueblo, New Mexico, Sampson Award Finalist.

Received for publication June 30, 2007; revisions received Oct 6, 2007; accepted for publication Oct 19, 2007.

Address for reprints: David D. Yuh, MD, Division of Cardiac Surgery, The Johns Hopkins Hospital, 600 North Wolfe Street, Blalock 618, Baltimore, MD 21287 (E-mail: dyuh@csurg.jhmi.jhu.edu).

J Thorac Cardiovasc Surg 2008;135:503-11 $0022-5223 / \$ 34.00$

Copyright (C) 2008 by The American Association for Thoracic Surgery

doi:10.1016/j.jtcvs.2007.10.042
Objective: The optimal timing for coronary artery bypass grafting after acute myocardial infarction is not well established. The California Discharge Database facilitates the study of this issue by providing data from a large patient cohort free of institutional bias. We examine the timing of coronary artery bypass grafting after acute myocardial infarction on short-term outcomes.

Methods: We reviewed California Discharge Data to identify 40,159 patients who were hospitalized for acute myocardial infarction (day 0 ) and underwent subsequent coronary artery bypass grafting. Patients were stratified by the timing of coronary artery bypass grafting to "early" (days 0-2) and "late" groups (day 3 or later). The primary outcome variable was all-cause hospital mortality. Multiple logistic and linear regression and propensity analyses assessed the risk of adverse events, controlling for factors associated with preoperative clinical acuity, including the Charlson Comorbidity Index, shock, mechanical ventilation, and the use of intra-aortic balloon counterpulsation.

Results: Of 9476 patients identified, $4676(49 \%)$ were in the early coronary artery bypass grafting group and $4800(51 \%)$ were in the late coronary artery bypass grafting group. A total of 444 patients $(4.7 \%)$ died during hospitalization, with a peak mortality rate of $8.2 \%$ among patients undergoing coronary artery bypass grafting on day 0 , declining to a nadir of $3.0 \%$ among patients undergoing coronary artery bypass grafting on day 3 . The mean time to coronary artery bypass grafting was 3.2 days. Patients undergoing early coronary artery bypass grafting experienced a higher mortality rate than those undergoing late coronary artery bypass grafting $(5.6 \%$ vs $3.8 \%, P<.001)$. Early coronary artery bypass grafting was an independent predictor of mortality after controlling for clinical acuity and on propensity analysis (odds ratio 1.43, $P=.003$ ).

Conclusion: Patients undergoing coronary artery bypass grafting within 2 days of hospitalization for acute myocardial infarction experienced higher mortality rates than those undergoing coronary artery bypass grafting 3 or more days after acute myocardial infarction, independently of clinical acuity. This suggests that coronary artery bypass grafting may best be deferred for 3 or more days after admission for acute myocardial infarction in nonurgent cases.

$\mathrm{M}$ uch attention has focused on the outcomes of coronary artery bypass grafting (CABG) in the setting of acute myocardial infarction (AMI). ${ }^{1-3} \mathrm{Al}-$ though it seems clear that patients who undergo CABG after AMI possess a higher risk of short-term mortality compared with patients who undergo elective $\mathrm{CABG}$, the optimal timing of surgical revascularization remains controversial. ${ }^{1,4-6}$ Recent randomized controlled trials and meta-analyses performed in the setting of AMI have convincingly demonstrated that primary percutaneous coronary interventions (PCIs) yield superior results to thrombolytic therapy with decreased rates of death, reinfarction, and stroke. ${ }^{7-9}$ Consequently, an increased number of patients 

Abbreviations and Acronyms
AMI = acute myocardial infarction
$\mathrm{CABG}=$ coronary artery bypass graft
IABP = intra-aortic balloon pump
ICD-9-CM = International Classification of Diseases,
Ninth Revision, Clinical Modification
$\mathrm{OR} \quad=$ odds ratio
PCI = percutaneous coronary intervention

are receiving coronary angiography in the setting of AMI. ${ }^{10,11}$ This, in turn, has led to an increase in identification of candidates for surgical coronary revascularization (ie, in the setting of failed angioplasty or left main/multivessel disease). With this focus on CABG in the context of AMI and new trends in early management, the optimal timing of CABG after AMI should be scrutinized.

Although no definitive recommendation exists regarding the appropriate timing of CABG after recent AMI, the notion that these patients assume greater risk for short-term mortality is gaining consensus. ${ }^{5,12-14}$ This is not surprising given that the majority of patients who undergo early CABG present with a higher degree of clinical acuity, which, in turn, translates to higher mortality rates. For the stable patient post-AMI for whom the culprit lesion has been effectively treated with PCI, it is common practice to discharge the patient to recover for some time before undergoing $\mathrm{CABG}$ electively. Less certain, however, is the optimal management of patients post-AMI who cannot be discharged from the hospital before CABG because of a tenuous PCI result, disease severity, unstable angina, or compromised ventricular function. The optimal timing for $\mathrm{CABG}$ in these patients post-AMI was the focus of this study in which we performed a retrospective review with multivariable and propensitybased adjustments. We hypothesize that by controlling for both clinical acuity and early surgical propensity, an optimal time interval between AMI and CABG can be identified such that early postoperative mortality is reduced.

\section{Materials and Methods Data Source}

The State of California Office of Statewide Health Planning and Development (Healthcare Quality and Analysis Division) provided hospital discharge data for the years 1999 to 2005. The State of California requires all licensed hospitals to submit data on all discharged patients every 6 months. These records thus comprise a $100 \%$ sampling of California nonfederal hospital discharges. All data are de-identified and include demographic information, including age, gender, race, information on primary and concomitant diagnoses recorded and procedures performed, discharge information (eg, disposition to skilled nursing facility, death), and administrative information (eg, costs, payer information, length of stay, level of care). Unlike many other databases, the California discharge database discriminates between prior and new diagnoses with respect to each hospital admission and identifies the dates of procedures and operations performed. These features facilitated the examination of outcomes related to the timing of CABG after AMI. Because individual patients are not identified in this multicenter registry report, the need for consent and institutional review board approval is waived at The Johns Hopkins Medical Institutions.

\section{Study Design}

A retrospective review of California discharge data was performed for the years 1999 to 2005 . We identified all adult patients $(>17$ years of age) who were admitted to a California hospital with the primary diagnosis of AMI as identified by International Classification of Diseases, Ninth Revision, Clinical Modification (ICD-9-CM) diagnosis codes (410.x). ${ }^{15}$ We combined these patients with those who underwent $\mathrm{CABG}$ of any type during their hospitalization (also identified by ICD-9-CM procedure codes 36.x).

Patients were excluded if they had valvular pathology (ie, stenosis, insufficiency or active endocarditis of the mitral, aortic, pulmonary, or tricuspid valves) or underwent any concomitant cardiac surgical procedures such as mitral or aortic valve repair or replacement, ventricular remodeling, or ventricular assist device placement. In addition, patients were excluded if they presented with "do not resuscitate" status on admission or if they were transferred from another hospital (because we did not have information on the timing of AMIs in these patients) (Table E1).

\section{Creation of Variables}

Variables crucial to the analysis not present in the data set were derived using existing data set variables and known ICD-9-CM codes. The primary derived variable was the timing of CABG after AMI. The data set parameters yielded time intervals expressed in "days" rather than finer time intervals of hours or minutes. In this analysis, we designated day 0 as the initial day of hospital admission. Baseline independent variables describing medical status and outcome variables (other than death) were developed from ICD9-CM coding and are subsequently listed in Table E2. We used the Charlson scoring system ${ }^{16}$ with Deyo adaptation ${ }^{17}$ as a standardized index reflecting the overall burden of comorbidities in the study population. Our primary outcome variable was all-cause in-hospital mortality.

\section{Patient Groups}

Because of the lack of standardized timing intervals for the performance of CABG after AMI, we grouped patients into "early" and "late" groups on the basis of the median time to CABG for our study population (day 3). Early CABG was thus defined as CABG performed on hospitalization day 0,1 , or 2 , and late $\mathrm{CABG}$ was defined as $\mathrm{CABG}$ occurring on or after day 3 . We also examined differences in outcomes dichotomized between other time points such as day 0 versus day 1 and after, day 0 and 1 versus day 2 and after, and so forth.

\section{Statistical Analysis}

Comparisons of baseline characteristics between study groups were performed using the Student $t$ test for continuous variables and the chi-square test for categoric variables. Mortality was first assessed for all risk factors using a univariate model. Significant predictors of mortality (both chronic and acute) were incorporated into 
a multivariable logistic regression model in a stepwise fashion to assess the effect of timing of CABG on mortality. Operative timing and mortality were plotted for all time points present to determine whether an optimal time for the performance of CABG after AMI exists.

To assess selection bias not controlled for in our multivariate model and to counter the censoring that occurs from early mortality, we used a propensity-adjustment model. We developed propensity scores (based on the likelihood of receiving early CABG on days 0,1 , or 2 ) derived from a logistic regression model incorporating 35 potential predictors of operative timing (Table E3). We incorporated the resultant propensity scores into a logistic regression model to negate selection bias in examining the effect of propensity for early CABG on in-hospital mortality. We also used quintile stratification for both internal validation of our technique and examination of the effects of timing in different subsets of propensity.

All odds ratios (ORs) are presented with $95 \%$ confidence intervals. All statistical analysis was performed with the aid of STATA software (version 9.0, StataCorp LP, College Station, Tex).

\section{Results}

Between 1999 and 2005, California hospitals recorded a total of 443,069 patient discharges with the primary diagnosis of AMI. Of these patients, 40,159 underwent CABG during their hospitalization. After exclusion of children $(<18$ years of age), hospital transfers, patients with concomitant cardiac procedures, and patients with incomplete data, 9476 patients were included in the final study population.

We noted that $75 \%$ of CABG $(\mathrm{n}=7086)$ procedures were performed during the first 5 days of hospitalization (Figure 1). The median time to CABG was 3 days (mean $3.2 \pm 3.0$ ). By stratifying according to this median, 4676 patients $(49 \%)$ were classified as the early $\mathrm{CABG}$ group (CABG performed on days 0,1 , or 2 ) and 4800 patients were classified as the late group (CABG performed on day 3 or later). The early group consisted of a lower percentage of female patients compared with the late group $(28 \%$ vs $33 \%, P<.001)$. Both groups had similar baseline comorbidities with mean Charlson scores of 5.2 and 5.8, respectively $(P<.001)$ (Table 1$)$. As expected, patients in the early CABG group were of higher acuity with greater proportions presenting with shock and requiring intra-aortic balloon pump (IABP) counterpulsation. Of note, the early group had a lower percentage of subendocardial infarcts $(45 \%$ vs $61 \%, P<.001)$.

In-hospital mortality for the entire study period was $4.7 \%$ (444 patients). Patients undergoing CABG on their initial day of admission had an $8.2 \%$ mortality rate. Plotting of mortality over time revealed a nadir for patients undergoing CABG on hospital day 3 with a mortality rate of $3.0 \%$ (Figure 1). This low mortality rate persisted for those receiving $\mathrm{CABG}$ on days 4 and 5 but increased thereafter. Patients who underwent CABG between days 14 and 28 experienced a $12.5 \%$ in-hospital mortality rate $(P<.01)$. The greatest decline in mortality in a 1-day period occurred for patients undergoing $\mathrm{CABG}$ on the first hospital day. A $3.7 \%$ decline in mortality $(P<$ $.05)$ was observed when CABG was performed on hospital day 1 compared with the day of admission.

Univariate analysis revealed early CABG to be highly associated with an increased risk of mortality (5.6\% vs $3.8 \%, P<.001)$. This positive association was confirmed with multivariable logistic regression analysis controlling for gender, baseline comorbidities as assessed by the Charlson index, and markers of clinical acuity, including IABP, shock, cardiac arrest on admission, and ventilation before CABG (Table 2). The OR of 1.43 corresponds to a $43 \%$ increase in the risk of death among patients who underwent early $\mathrm{CABG}$, controlling for the aforementioned markers of clinical acuity. Other strong predictors of death included IABP, shock on admission, cardiac arrest on admission, female gender, and Charlson index.

After we controlled for the propensity to undergo early surgery (C-index 0.76), CABG performed before day 3 continued to be positively associated with mortality risk (OR

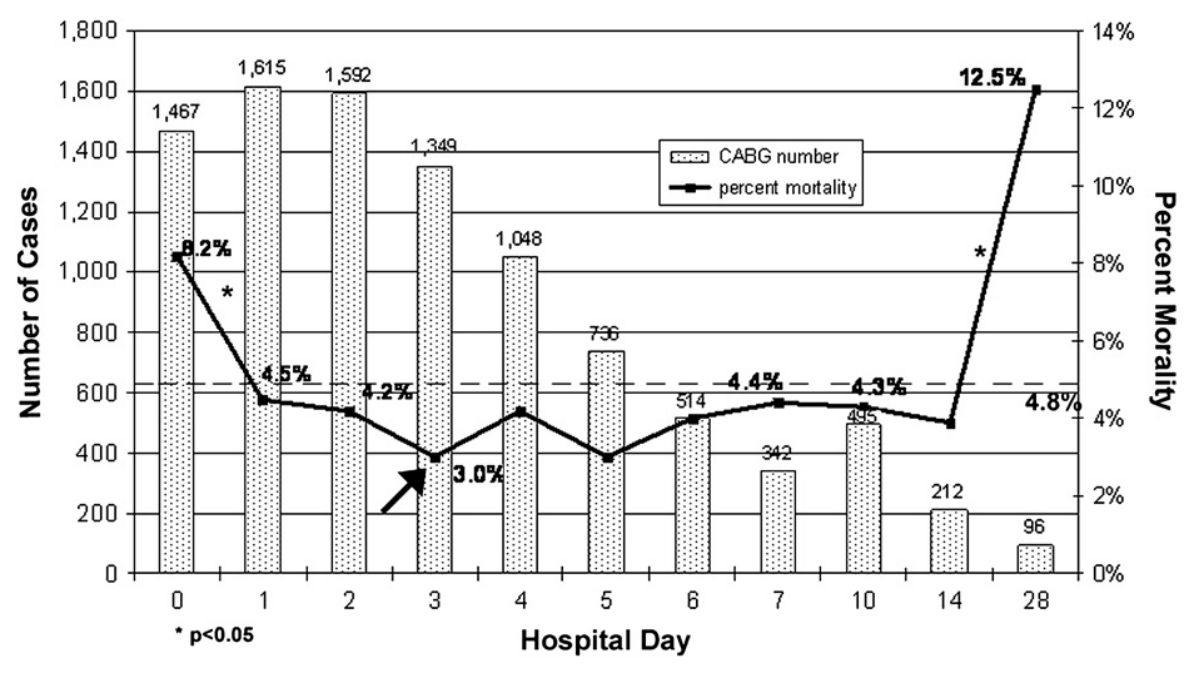

Figure 1. CABG volume and mortality over time. Distribution of CABG volume (left Y-axis, number of cases per day) and percent mortality (right Y-axis) per day of hospitalization. Dashed horizontal line represents total mortality over the study period (4.8\%). Black arrow points to nadir of mortality occurring on day 3. CABG, Coronary artery bypass graft. 
TABLE 1. Baseline demographics and acuity between early and late coronary artery bypass graft groups

\begin{tabular}{|c|c|c|c|}
\hline & $\begin{array}{c}\text { Early CABG } \\
N=4676\end{array}$ & $\begin{array}{c}\text { Late CABG } \\
N=4800\end{array}$ & $\begin{array}{c}P \\
\text { value* }\end{array}$ \\
\hline \multicolumn{4}{|c|}{$\mathrm{N}(\%)$ or mean (SD) } \\
\hline Mean age (SD) & $66.6(11)$ & $68.6(11)$ & $<.001$ \\
\hline Female $(\%)$ & $1306(28)$ & $1566(33)$ & $<.001$ \\
\hline $\begin{array}{l}\text { Black, Hispanic, Native } \\
\text { American, Asian/Pacific } \\
\text { Islander } \pm\end{array}$ & $930(20)$ & $1312(27)$ & $<.001$ \\
\hline Obesity & $623(13)$ & $602(13)$ & .26 \\
\hline Charlson index (SD) & $5.2(1.8)$ & $5.8(1.9)$ & $<.001$ \\
\hline Subendocardial infarct & $2114(45)$ & $2927(61)$ & $<.001$ \\
\hline $\begin{array}{l}\text { Cardiac arrest on } \\
\text { admission }\end{array}$ & $65(1)$ & $78(2)$ & .35 \\
\hline Shock & $314(7)$ & $166(3.4)$ & $<.001$ \\
\hline IABP before CABG & $469(10)$ & $412(8.6)$ & .02 \\
\hline V-Fib on admission & $81(1.8)$ & $91(1.9)$ & .78 \\
\hline A-Fib on admission & $570(12)$ & $774(16)$ & $<.001$ \\
\hline Angiogram before $C A B G$ & $4213(90)$ & $4592(96)$ & $<.001$ \\
\hline $\begin{array}{l}\text { Coronary stent } \\
\text { before CABG }\end{array}$ & $493(11)$ & $457(10)$ & .1 \\
\hline Bypass (4 vessels) & $1261(27)$ & $1245(26)$ & .26 \\
\hline Bypass (3 vessels) & $1552(33)$ & $1711(36)$ & .01 \\
\hline Bypass (2 vessels) & $1319(28)$ & $1288(27)$ & .13 \\
\hline Bypass (1 vessels) & $452(10)$ & $435(9)$ & .31 \\
\hline
\end{tabular}

$C A B G$, Coronary artery bypass graft; $S D$, standard deviation; $I A B P$, intraaortic balloon pump; $V$-Fib, ventricular fibrillation; $A$-Fib, atrial fibrillation. ${ }^{*} P$ value is based on comparison between 2 groups by either chi-square or Student $t$ test. \pm Race or ethnic group was a variable present in the data set.

$1.40, P=.03$ ) (Table 2). Propensity scores were associated with early CABG (mean score 0.61 vs $0.37, P<.001$ ) confirming that they did, in fact, predict early surgery. When stratified into propensity quintiles, only the lowest propensity group retained this positive association. Therefore, those patients with the least need to undergo early $\mathrm{CABG}$ had a 2 -fold risk of death if they underwent early CABG (OR 1.58, $P=$ .03). By performing serial propensity-adjusted analyses for all combinations of early and late $\mathrm{CABG}$, we found that the relationship between early $\mathrm{CABG}$ and increased mortality ceased on hospital day 4 (OR for death if CABG performed on days $0-3$ vs $\geq 4,0.92, P=.45$ ). Thus, hospital day 3 seemed to be the cutoff point whereby further delay to CABG did not improve survival.

To determine optimal operative timing in patients with AMI presenting with high clinical acuity, we examined those patients with transmural infarctions (ie, acute infarctions not coded as "subendocardial") who were classified as having shock or who underwent IABP placement. Among these patients, we noted that the lowest mortality rates were observed when CABG was performed on hospital day 3 (Figure 2). As for the entire study population, mortality rates increased after day 4 among patients in this high-acuity group. The overall
TABLE 2. Risk factors for mortality determined from multivariable logistic regression analysis

\begin{tabular}{|c|c|c|}
\hline Risk factor & $\begin{array}{c}\text { OR } \\
(95 \% \mathrm{Cl})\end{array}$ & $\begin{array}{c}P \\
\text { value* }\end{array}$ \\
\hline \multicolumn{3}{|c|}{ Multivariable analysis without propensity adjustment } \\
\hline Day of CABG (across entire study period) & $0.99(0.95-1.02)$ & .65 \\
\hline Early CABG (days $0-2$ ) & $1.43(1.12-1.18)$ & .003 \\
\hline Female gender & $1.46(1.16-1.85)$ & .002 \\
\hline Charlson index & $1.29(1.20-1.39)$ & $<.001$ \\
\hline Shock & $3.58(2.41-5.32)$ & $<.001$ \\
\hline Cardiac arrest on admission & $3.23(1.59-6.54)$ & $<.001$ \\
\hline IABP before CABG & $1.51(1.09-2.1)$ & .01 \\
\hline Ventilated before CABG & $1.25(0.79-1.98)$ & .32 \\
\hline V-Fib on admission & $1.03(0.45-2.23)$ & .98 \\
\hline Anterolateral infarct & $1.08(0.47-2.29)$ & .93 \\
\hline
\end{tabular}

Multivariable analysis with propensity adjustment

\begin{tabular}{lcc}
\hline Early CABG (days 0-2) & $1.40(1.12-1.74)$ & .003 \\
Propensity score & $1.32(0.87-2.01)$ & .18 \\
\hline \multicolumn{2}{c}{ Risk of death with early CABG as stratified by propensity quintile } \\
\hline Quintile 1 (Pscore 0.03-0.32) & $1.58(1.05-2.34)$ & .03 \\
Quintile 2 (Pscore 0.32-0.36) & $1.21(0.75-1.94)$ & .43 \\
Quintile 3 (Pscore 0.39-0.46) & $1.46(0.83-2.58)$ & .19 \\
Quintile 4 (Pscore 0.46-0.60) & $1.39(0.77-2.5)$ & .28 \\
Quintile 5 (Pscore 0.60-0.90) & $3.80(0.42-34.3)$ & .23
\end{tabular}

$O R$, Odds ratio; $\mathrm{Cl}$, confidence interval; $C A B G$, coronary artery bypass graft; $V$-Fib, ventricular fibrillation; $I A B P$, intra-aortic balloon pump; Pscore, propensity score. This analysis was performed with and without propensity adjustment and with quintile propensity stratification. ${ }^{*} P$ value is based on multivariable logistic regression analysis, using all factors significant on univariate analysis.

in-hospital mortality rates for patients presenting with shock and IABP placement were $24 \%$ (90/480 patients) and $7 \%$ (65/881 patients), respectively.

\section{Discussion}

Although the debate regarding the optimal timing of CABG after AMI is not yet settled, the findings of this study lend support to an increasing body of medical literature demonstrating increased risks of urgent surgical coronary revascularization in the setting of AMI. As in many issues in cardiac surgery, this topic has undergone evolution as technology has improved and mortality rates for CABG have declined. Studies conducted in the early 1980s by DeWood and colleagues ${ }^{18,19}$ focusing on this question showed a benefit to performing early CABG. Although valid at the time, these studies were conducted in an era before the widespread institution of PCI and thrombolysis in the setting of AMI.

More recently, retrospective examinations have concluded that CABG should be deferred, when possible, for 3 or more days after AMI. ${ }^{12,13,20}$ Lee and colleagues ${ }^{13,20}$ have conducted several studies describing the appropriate timing of CABG after AMI. By using the state of New 


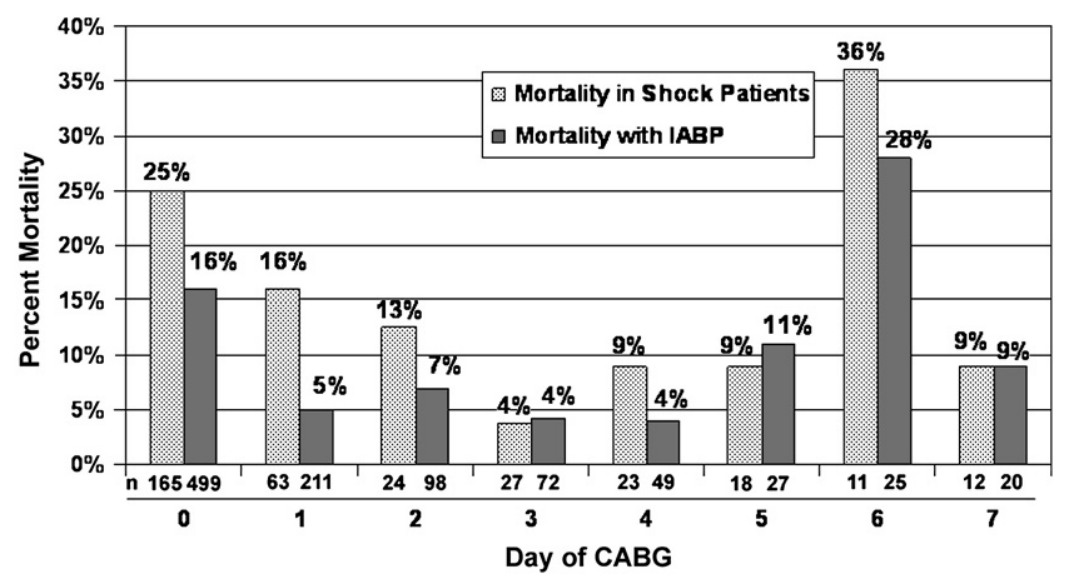

Figure 2. Mortality in patients undergoing acute CABG. Percent mortality for those patients with transmural AMI (defined as acute infarctions not coded as "subendocardial") and shock (light grey bars) or IABP placement preoperatively (dark grey bars). Note that for both patient sets, the nadir of mortality occurs on hospital day 3. Total patient numbers for each day (n) are given below each bar. IABP, Intra-aortic balloon pump; $C A B G$, coronary artery bypass graft.

York database, the authors showed that the risk of early CABG is substantially higher before hospital day 3, with a doubling of mortality risk compared with patients who underwent later surgery. This positive association between early CABG and mortality was seen to be particularly important for transmural infarcts. Another study by Voisine and associates $^{12}$ concluded that CABG is best deferred for a period of 7 days after AMI. One should note, however, that this study examined only 77 subjects from a single institution (1991-2005) who underwent CABG within 24 hours of admission.

Although these prior studies are valuable in supporting the notion that delayed $\mathrm{CABG}$ is preferable under elective clinically stable circumstances, they have not specifically addressed patients with AMI presenting with higher clinical acuity. Kamohara and colleagues ${ }^{14}$ attempted to examine this issue by focusing on patients who underwent urgent CABG only. In their report, they conclude that patients who underwent early CABG (ie, within 6 hours of AMI) experienced higher mortality rates $(9.1 \%$ vs $2.9 \%)$ than those who underwent CABG later in their course (ie, 6-24 hours after AMI). Although this study attempted to examine the effect of timing among acutely ill patients, it is a single-center report that did not include patients undergoing CABG 24 or more hours after AMI. It is possible that outcomes would have improved among these patients if $\mathrm{CABG}$ had been further delayed.

In our analysis, we attempted to bridge this gap in knowledge by focusing on hospitalized patients, identifying a modern cohort of patients among multiple centers who presented with higher clinical acuity than those reported in prior studies but did not require urgent $\mathrm{CABG}$. We used this strategy to determine the optimal timing of surgery among patients with AMI who underwent CABG during their initial hospitalization.

Our study uniquely possesses an even distribution of postAMI CABG timing intervals. With 1477 patients undergoing $\mathrm{CABG}$ on their initial hospital day, early $\mathrm{CABG}$ was well represented in this sample. The fact that we only examined patients who required hospital admission may partially explain why the in-hospital mortality rate of $4.8 \%$ may be higher than previously reported mortality rates, typically ranging from $2.3 \%$ to $3.3 \%$. $^{12,20}$

This study is also distinguished by the robust statistical methods we used to control bias inherent in its retrospective design. We addressed 2 important statistical problems. First, patients who underwent early CABG inherently assumed a greater mortality risk associated with their increased level of acuity, constituting a selection bias. Although traditional multivariate models can attempt to limit this risk, an obvious flaw is the difficulty of accounting for all potential confounders. Second, patients who underwent early CABG and died are excluded from being a part of the late CABG group. This effect, termed censoring, leads to relative inequality between the 2 groups and violates a fundamental assumption of multivariate models. Because those patients who die cannot be part of the late group, a bias that inflates late group survival occurs. In the absence of a gold standard blinded, randomized controlled trial, these effects are difficult to control.

We attempted to address these statistical concerns by using a propensity-adjusted analysis. The use of propensity scores represents another tool to help eliminate bias in a retrospective study and has been used successfully in other landmark surgical studies. ${ }^{21-23}$ The propensity score (ranging from 0-100) predicts which patients are likely to receive a treatment or, in this case, early CABG. Propensity scores are particularly useful when the primary outcome is rare. Unlike traditional multivariate analyses, the propensity-based approach addresses the issues of selection bias and censoring by adjusting for the likelihood of receiving early CABG, not simply by adjusting for known confounders. We think that the use of a propensity-based approach is an effective way to address the issue of timing in this analysis.

By using propensity-adjusted analysis, the findings of this study are consistent with previous reports showing that early CABG (before day 3) is associated with an increased risk of 
mortality. We found an approximate doubling of mortality among those patients who underwent CABG before day 3 (5.6\% vs $2.8 \%$ ). This effect was independent of factors associated with acuity and propensity-based analysis. It is noteworthy that on quintile assessment, the effect seemed to be restricted to the lowest propensity quintile. In other words, those patients with low likelihood for undergoing early $\mathrm{CABG}$ derived the worst effect from receiving early CABG. These results suggest that early elective CABG in the setting of AMI is associated with a heightened postoperative mortality risk that might otherwise be ameliorated by deferring the operation. Our analysis suggests that 3 to 5 days seems to be an optimal timing window for performing an elective CABG after AMI.

An unexpected observation from this study showed that postoperative mortality rates trended upward after day 5 . In fact, patients who underwent CABG between days 14 and 28 experienced a $12.5 \%$ risk of mortality. In contrast, previous studies have noted a steady downward trend in mortality with increasing time after AMI. In our cohort, among patients who underwent $\mathrm{CABG}$ after day $14,16 \%$ presented in shock $(\mathrm{n}=7), 29 \%$ required mechanical ventilation $(\mathrm{n}=13)$, and $20 \%$ required IABP placement $(n=9)$ preoperatively. Thus, it is likely that our findings are reflective of sicker patients who required extended hospitalization after an AMI.

We also examined a critically ill population by examining only those patients with a transmural infarction who presented in hemodynamic shock or who underwent IABP placement. It is noteworthy that these patients also derived mortality benefit from waiting until day 3 to perform CABG but experienced increased mortality if CABG was performed after day 4 . These findings support that, even in the setting of high-acuity patients, survival benefit may be derived from deferring CABG 2 to 3 days after AMI.

\section{Use of the California Database}

We chose to use the California statewide database in this analysis because of 2 key features. The first was the ability to gauge procedural timing, which was crucial to the analysis. The second was the discrimination between diagnoses present on admission to the hospital versus new diagnoses made during the hospitalization. This allowed us to determine whether a patient characteristic was preexistent or developed subsequent to CABG. For the purposes of retrospective analyses, a large database provides a large sample size that is free of institutional bias. Because individual institutional practices with respect to performing $\mathrm{CABG}$ after AMI differ dramatically, we think that this freedom from institutional bias gives our study added power.

\section{Study Limitations}

We recognize that there are several limitations in our study. First, our study is retrospective and cannot account for inherent undocumented differences in patient characteristics.
Although we attempted to control for selection bias with propensity and multivariate statistical methods, we concede that a fundamental problem of any retrospective study examining interventional timing is the inherent heightened acuity of patients who undergo early CABG. Second, we were reliant on the variables provided by the California discharge database. Several markers of clinical acuity (eg, left ventricular ejection fraction) were not available and thus could not be included. Finally, administrative databases, including the one we used, and studies based on them are reliant on accurate coding. We acknowledge that the data presented here were not necessarily entered by individuals with clinical expertise. Subtle differences in coding definitions may exist between different institutions; neither the coding nor procedural timing included in the California discharge data has been validated for patients undergoing CABG. However, although errors and variance in the data undoubtedly exist, we have assumed that these are randomly distributed and should not lead to significant bias in our conclusions. Without a randomized controlled clinical trial, we thought that a retrospective study with compensatory statistical methods constituted a reasonable approach to address this issue.

\section{Why Delayed CABG May Be Preferable}

In light of the present findings and similar conclusions by others, we must ask the question of why delayed CABG leads to improved outcomes in the setting of AMI. Intuitively it seems that early reperfusion would lead to the preservation of myocardium, thus limiting infarct size. However, reperfusion injury can lead to increased damage to the vital myocardium beyond the ischemic insult. ${ }^{24}$ Furthermore, it is known that during the acute phases of an infarct, whole body inflammatory states are increased with increased levels of C-reactive protein as a marker. ${ }^{25,26}$ It is possible that reperfusioninduced inflammatory states have systemic manifestations that increase mortality. Beyond biological explanations, on a programmatic level, it is also possible that outcomes are improved by operating in a planned and controlled setting where routine physician, nursing, and ancillary staff are present and rested. Whether these or other unknown factors contribute to reduced mortality by deferring CABG after AMI is still unclear and a subject of considerable interest.

\section{Conclusions}

In this analysis, we sought to determine the optimal CABG timing after AMI among patients who underwent surgery during the index hospitalization. We observed a reduced mortality rate when CABG was deferred until hospital day 3 , consistent with previous reports. This effect was also observed among high-acuity patients who presented in hemodynamic shock or requiring IABP support. These results suggest that CABG may best be deferred for 3 or more days after admission for AMI under nonurgent clinical circumstances. 
Dr Weiss is the Irene Piccinini Investigator in Cardiac Surgery. Dr Nwakanma is a Hugh R. Sharp Cardiac Surgery Research Fellow. The authors thank the State of California Office of Statewide Health Planning and Development for the provision of data.

\section{References}

1. Coleman WS, DeWood MA, Berg R Jr, Selinger SL, Leonard JJ, Siwek LG. Surgical intervention in acute myocardial infarction: an historical perspective. Semin Thorac Cardiovasc Surg. 1995;7: 176-83.

2. Solodky A, Behar S, Boyko V, Battler A, Hasdai D. The outcome of coronary artery bypass grafting surgery among patients hospitalized with acute coronary syndrome: the Euro Heart Survey of acute coronary syndrome experience. Cardiology. 2005;103:44-7.

3. Zaroff JG, diTommaso DG, Barron HV. A risk model derived from the National Registry of Myocardial Infarction 2 database for predicting mortality after coronary artery bypass grafting during acute myocardial infarction. Am J Cardiol. 2002;90:1-4.

4. Hill JD, Kerth WJ, Kelly JJ, Selzer A, Armstrong W, Popper RW, et al. Emergency aortocoronary bypass for impending or extending myocardial infarction. Circulation. 1971;43(5 Suppl):I105-10.

5. Creswell LL, Moulton MJ, Cox JL, Rosenbloom M. Revascularization after acute myocardial infarction. Ann Thorac Surg. 1995;60: 19-26.

6. Gardner TJ, Stuart RS, Greene PS, Baumgartner WA. The risk of coronary bypass surgery for patients with postinfarction angina. Circulation. 1989;79(6 Pt 2):I79-80.

7. Keeley EC, Boura JA, Grines CL. Comparison of primary and facilitated percutaneous coronary interventions for ST-elevation myocardial infarction: quantitative review of randomised trials. Lancet. 2006;367: 579-88.

8. Cucherat M, Bonnefoy E, Tremeau G. Primary angioplasty versus intravenous thrombolysis for acute myocardial infarction. Cochrane Database Syst Rev. (Online). 2003. CD001560.

9. Keeley EC, Boura JA, Grines CL. Primary angioplasty versus intravenous thrombolytic therapy for acute myocardial infarction: a quantitative review of 23 randomised trials. Lancet. 2003;361:13-20.

10. Cook S, Walker A, Hugli O, Togni M, Meier B. Percutaneous coronary interventions in Europe: prevalence, numerical estimates, and projections based on data up to 2004. Clin Res Cardiol. 2007;96: 375-82.

11. Anderson HV, Shaw RE, Brindis RG, Hewitt K, Krone RJ, Block PC, et al. A contemporary overview of percutaneous coronary interventions. The American College of Cardiology-National Cardiovascular Data Registry (ACC-NCDR). J Am Coll Cardiol. 2002;39:1096-103.

12. Voisine P, Mathieu P, Doyle D, Perron J, Baillot R, Raymond G, et al. Influence of time elapsed between myocardial infarction and coronary artery bypass grafting surgery on operative mortality. Eur J Cardiothorac Surg. 2006;29:319-23.

13. Lee DC, Oz MC, Weinberg AD, Lin SX, Ting W. Optimal timing of revascularization: transmural versus nontransmural acute myocardial infarction. Ann Thorac Surg. 2001;71:1197-204.

14. Kamohara K, Yoshikai M, Yunoki J, Fumoto H, Hamada M, Murayama J, et al. Surgical revascularization for acute coronary syndrome: comparative surgical and long-term results. Jpn J Thorac Cardiovasc Surg. 2006;54:95-102.

15. International Classification of Diseases, Ninth Revision. Washington, DC: Public Health Service, US Department of Health and Human Services: 1988

16. Charlson ME, Pompei P, Ales KL, MacKenzie CR. A new method of classifying prognostic comorbidity in longitudinal studies: development and validation. J Chron Dis. 1987;40:373-83.

17. Romano PS, Roos LL, Jollis JG. Adapting a clinical comorbidity index for use with ICD-9-CM administrative data: differing perspectives. J Clin Epidemiol. 1993;46:1075-9; discussion 81-90.

18. DeWood MA, Heit J, Spores J, Berg R Jr, Selinger SL, Rudy LW, et al. Anterior transmural myocardial infarction: effects of surgical coronary reperfusion on global and regional left ventricular function. $J$ Am Coll Cardiol. 1983;1:1223-34.
19. DeWood MA, Spores J, Berg R Jr, Kendall RW, Grunwald RP, Selinger SL, et al. Acute myocardial infarction: a decade of experience with surgical reperfusion in 701 patients. Circulation. 1983;68(3 Pt 2): II8-16.

20. Lee DC, Oz MC, Weinberg AD, Ting W. Appropriate timing of surgical intervention after transmural acute myocardial infarction. J Thorac Cardiovasc Surg. 2003;125:115-9; discussion 9-20.

21. Adamina M, Guller U, Weber WP, Oertli D. Propensity scores and the surgeon. Br J Surg. 2006;93:389-94.

22. Connors AF Jr, Speroff T, Dawson NV, Thomas C, Harrell FE Jr, Wagner $\mathrm{D}$, et al. The effectiveness of right heart catheterization in the initial care of critically ill patients. SUPPORT Investigators. JAMA. 1996;276:889-97.

23. Mangano DT, Tudor IC, Dietzel C. The risk associated with aprotinin in cardiac surgery. $N$ Engl J Med. 2006;354:353-65.

24. Roberts C, Schoen FJ, Kloner RA. Effects of coronary reperfusion on myocardial hemorrhage and infarct healing. Am J Cardiol. 1983;52: 610-4.

25. Anzai T, Yoshikawa T, Shiraki H, Asakura Y, Akaishi M, Mitamura H, et al. C-reactive protein as a predictor of infarct expansion and cardiac rupture after a first Q-wave acute myocardial infarction. Circulation. 1997;96:778-84.

26. Pietilä K, Harmoinen A, Pöyhönen L, Ruosteenoja R. C-reactive protein in subendocardial and transmural myocardial infarcts. Clin Chem. 1986; 32:1596-7.

\section{Discussion}

Dr Mitruka (Rancho Mirage, Calif). I think there is little question that early revascularization confers a survival advantage in patients with AMI. This has been demonstrated by the cardiologists performing PCI in this patient population. Indeed, the door to balloon time is now a benchmark for programmatic success. This early intervention has resulted, however, in many surgeons being cajoled or even coerced into performing high-risk operations earlier than may be beneficial. The optimal timing of surgical revascularization after AMI remains somewhat controversial, although it is generally accepted that waiting is better. This was demonstrated by the Columbia group evaluating New York State databases, and they showed that waiting to operate, especially in patients with transmural infarcts, results in better outcomes.

This retrospective study performed by the Johns Hopkins group using California discharge data adds to the growing body of literature that attempts to objectify the optimal timing of CABG after AMI. This group is to be congratulated for a statistical tour de force that overcame many of the inherent limitations and biases of a retrospective study in drawing meaningful conclusions. By using multiple logistic and linear regression, as well as propensity-adjusted multivariate analysis, the risk of adverse events could be assessed while controlling for factors associated with high preoperative clinical acuity. With this methodology, they were able to conclude that early CABG less than 3 days after an AMI was an independent predictor of mortality after controlling for clinical acuity and surgical propensity. Identifying the optimal timing of CABG after AMI to be 3 to 5 days to reduce postoperative mortality will be of clear clinical benefit. Furthermore, outlining that patients with higher acuity undergoing operation sooner will have higher morbidity will be beneficial in aligning outcome expectations between surgeons and our referring physicians. With that long-winded preamble, I have a few questions that you are invited to answer individually.

It is generally well accepted by most surgeons that preoperative assessment of left ventricular function, particularly after an AMI, is one of the best predictors of postoperative outcome. Yet, left 
ventricular function in this particular study as a variable was not assessed. Could you comment as to why?

Dr Weiss. I agree with you that left ventricular function is important. The reason we were not able to include it was because it was simply not a component of the California discharge database. Clearly these administrative databases have limitations, the principle one being a limitation of the data and variables that would be present if you had an opportunity to design the study from the beginning and oversee data collection. If we had control of the data set, we certainly would be interested in examining ventricular function. In addition, there is selection bias associated with retrospective administrative databases and an inability to recognize undocumented inherent patient differences.

Rather than focusing on the weaknesses of these studies, I like to focus on the strengths, being that these types of studies provide a broad multi-institutional sample. They allow examination of outcomes that are applicable to both small centers and large academic institutions. They offer another tool for providing evidence-based guidelines for our patients. I think the best way to address variables that are not present in the data set is to conduct a secondary study, perhaps using institutional data where you can control what variables are present. In fact, we are in the process of conducting a similar study based on our own institutional data with patients undergoing $\mathrm{CABG}$, and we hope that this will provide some of the answers that are not readily available using the California database.

Dr Mitruka. The anatomic location and size of the infarct are allegedly addressed by the comorbidity index, the Charlson index. However, it is also well recognized that patients with transmural infarcts that are perhaps anterior infarcts pose a higher risk in the perioperative period when operated on sooner rather than later. Was there any way to distinguish those patients in this database during your analysis to come up with a subgroup of patients who perhaps were at even higher risk?

Dr Weiss. We could identify the location of the infarct on the basis of the data we had. We incorporated that information into our propensity adjustment. On the multivariable logistic regression, we only used anterolateral infarct because that was the only factor that was a significant predictor of mortality on univariate analysis. So the answer to your question is yes, we did look at those variables. We incorporated them in a univariate model, and then we used them in our multivariable model accordingly.

Dr Mitruka. We, as clinical surgeons, oftentimes feel as though the longer we can wait, or we are taught to believe that the longer we can wait after an AMI before operating, the better, so I was a bit surprised to see that the longer you waited with patients, 2 to 3 weeks after an MI, the higher the mortality. We would have expected that the mortality decreases over the course of time. Would you comment on that?

Dr Weiss. As you pointed out, according to the studies done by the Columbia group, you would expect mortality to decrease over time. That has been shown in the past, and our findings would seem to contradict their findings. I think the difference lies in the fact that we looked at a subset of patients who were hospitalized and could not be discharged. Previous studies looked at patients who could be discharged. We thus examined a more acute cohort of patients, and I speculate that the reason some of these patients had higher mortality was because those who underwent operation later possessed higher clinical acuity with more comorbidities. This is perhaps the type of patient who has brittle diabetes, congestive heart failure, or COPD. This is the type of patient who you would prefer not to operate on, but for one reason or another later on in their hospitalization your hand is forced. We do have data to back that up. We examined that subset of patients, the late patients, and we did see that their Charlson Index was a full point higher than that of the remainder of the study population, so they indeed had a higher comorbidity index. In addition, those patients had a higher percentage of shock, higher percentage of intraaortic balloon pump, so I suspect that the difference is that our patient population is slightly different and that those patients operated on later were more acutely ill.

Member of the audience: I just want to ask one quick question. Did you look at the mortality rate for AMI without surgery? Is this an effect that occurs because the highest death rate from AMI is early, and this is an effect maybe of the disease rather than surgery?

Dr Weiss. That is a good point. We did not specifically do the same analysis on AMI without surgery. We designed the study to capture data such that we only looked at those who received surgery. I agree with you, and I think it is probably true that this is a function of the disease process, but within that disease process, if we look at the subset of those who have required surgery, we should still be able to identify the optimal time for CABG surgery in that cohort.

Dr Vallieres (Seattle, Wash). I am not a cardiac surgeon but I was wondering if you had looked at which day of the week (eg, Monday, Tuesday) when the infarct occurred and correlated to the day of the week when the revascularization occurred and whether this had an impact on these variables.

Dr Weiss. We did not. It was not present in the data set, and we did not look at it.

Dr Cohen. I had a couple of questions, but as the discussion ensued they turned into comments. The first comment is that I have to take issue with the statement that there are patients early on who would have fared better had you waited to operate. I think the study of your design might have been better had you excluded some of the patients who were absolutely without question going to die early without operation because I think they would have died had you waited, and so I think that invalidates that statement.

The second thing is that I have to emphasize what our primary discussant said. I think that ventricular function in the face of $\mathrm{AMI}$ and in determining when that patient is going to undergo operation is absolutely crucial, and I think that might be the fatal blow in determining the validity of this study because this study is incredibly important. Heart surgeons are going to rely on studies such as this in determining how to make life and death decisions for patients. I just don't know how you can do it without knowing what the ventricular function is in the face of an AMI.

Dr Weiss. As I said, we are attempting to perform a second study using our own institutional data that may answer some of those questions.

Dr Slater (Portland, Ore). I have some quick data questions. Did you segregate the data for men and women? Is the day of hospitalization the same as the day of MI for your group or was there any variability in that some patients come in several days after their MI? You have the date of surgery, but did you break it down by patients who came in, had an AMI on say Monday, Tuesday, are catheterized, and you are operating Tuesday night, versus patients who 
are catheterized and scheduled for surgery the following day, and they are done as an elective case?

Dr Weiss. We did not segregate on the basis of gender. We did look at gender in our multivariable model. Female gender was a predictor of worse outcomes, as has been shown previously.

The structure of the database does not really allow us to discriminate based on the hours at which you receive your CABG. It is only based on days. It is one of the limitations of the study, and we acknowledge that.
To answer your question about which patients presented and when they presented, these were all patients whose hospital day zero was their initial day of hospitalization, and it was also their day of presentation, the day that they had an AMI. We went to great efforts to identify patients who were admitted to the hospital solely with the principle diagnosis of AMI. For example, we excluded hospital transfers. We wanted to identify a group of patients for whom day zero truly was the day that they were admitted with AMI.

\section{UN THE MOVE?}

Don't miss a single issue of the journal! To ensure prompt service when you change your address, please photocopy and complete the form below.

Please send your change of address notification at least six weeks before your move to ensure continued service. We regret we cannot guarantee replacement of issues missed due to late notification.

\section{JOURNAL TITLE:}

Fill in the title of the journal here.

\section{OLD ADDRESS:}

Affix the address label from a recent issue of the journal here.

\section{COPY AND MAIL THIS FORM TO:}

Elsevier Inc.

Subscription Customer Service

6277 Sea Harbor Dr

Orlando, FL 32887

\section{NEW ADDRESS:}

Clearly print your new address here.

Name

Address

City/State/ZIP

OR FAX TO:

407-363-9661

OR E-mail:

elspcs@elsevier.com
OR PHONE:

800-654-2452

Outside the U.S., call

407-345-4000 
TABLE E1. Exclusion criteria for study

Exclusion criteria
ICD-9-CM codes

NA

NA

NA

$394,394.0,394.1,394.2,394.9,424.0$

$395,395.0,395.1,395.2,395.9,424.1,746.5,746.6$

$396,396.0,396.2,396.3,396.8,396.9,746.3,746.4$

$397.1,424.3,746.00,746.01,746.02,746.09$

$397,397.0,424.2,746.1$

$996.02,996.71$

$35.1,35.12,35.2,35.23,35.24,35.33,35.98,35.99$

$35.01,35.11,35.21,35.22$

$37.5,37.51$

$37.64,37.65,37.66$

$35.03,35.13$

$35.04,35.14$
No. of patients excluded (original $\mathrm{N}=\mathbf{4 0 , 1 5 9 \text { ) }}$

1

15,276

13,261

1108

439

184

2

69

1

15

11

0

318

Pulmonary valve replacement/repair

Tricuspid valve replacement/repair

Final study population

ICD-9-CM, International Classification of Diseases, Ninth Revision, Clinical Modification; NA, not available. 
TABLE E2. Independent and dependent variables generated from ICD-9 and ICD-9-CM coding

\begin{tabular}{|c|c|}
\hline \multicolumn{2}{|c|}{ Primary variable day of CABG } \\
\hline \multicolumn{2}{|l|}{ Baseline } \\
\hline Independent variables & ICD-9-CM codes used \\
\hline Charlson index* & See below \\
\hline Diabetes mellitus (Y/N) & $250,250.0250 .1,250.3,250.7,250.8,250.9$ \\
\hline COPD & $491.2,491.20,491.21,491.22$ 492, 492.0, 492.8 \\
\hline Hypertension & $401,401.1,401.91$ \\
\hline CRF & 585,588 \\
\hline History of stroke & $431,433,433.3,433.8,433.9,434,434.0,434.1,434.9$ \\
\hline Hyperlipidemia & $272.2,272.4$ \\
\hline Obesity & $278.0,278.00,278.01$ \\
\hline PVD & $443,443.8,443.89,443.9$ \\
\hline \multicolumn{2}{|l|}{ Type of AMI: } \\
\hline Anterolateral & $410,410.0,410.00,410.01,410.02,410.1410 .10,410.11,410.12$ \\
\hline Posterior & $410.6,410.60,410.61,410.62$ \\
\hline Inferior & $410.4,410.40,410.41,410.42$ \\
\hline Lateral & $410.5,410.50,410.51,410.52$ \\
\hline Subendocardial & $410.7,410.70,410.71,410.72$ \\
\hline Acuity variables & ICD-CM (for procedures) codes \\
\hline CPR & 99.63 \\
\hline IABP before CABG & 376.1 \\
\hline Conduction disorder on admission & $\begin{array}{l}426,426.0,426.1,426.10,426.11,426.12,426.13,426.2,426.3,426.4, \\
\quad 426.5,426.50,426.51,426.52,426.53,426.54,426.6,426.8,426.81, \\
426.89,426.9\end{array}$ \\
\hline Ventricular fibrillation on admission & $427.4,427.41,427.42$ \\
\hline Cardiac arrest on admission & $427.5,779.85$ \\
\hline Mechanical ventilation before CABG & $967,967.0,967.1,967.2,960.4$ \\
\hline Shock & 785.51 \\
\hline Atrial fibrillation on admission & $427.3,427.31,427.32$ \\
\hline Ventricular tachycardia on admission & 427.1 \\
\hline Coronary stent before CABG & $00.45,00.46,00.47,00.48,00.66,36.0136 .02,36.05,36.06,36.07$ \\
\hline Angiogram before CABG & $88.5,88.50,88.51,88.52,88.53,88.54,88.55,88.56,88.57$ \\
\hline
\end{tabular}

\begin{tabular}{ll}
\hline \multicolumn{1}{c}{ Outcome variables } & \\
\hline Postoperative LOS & Present in database \\
Postoperative stroke & $431,433,433.3,433.8,433.9,434,434.0,434.1,434.9$ \\
Prolonged ventilation $(>96 \mathrm{~h})$ & 967.2 \\
Postoperative ARF & $584,584.6,584.7,584.8,584.9,586$ \\
& $587,588,5880,588.1,588.8,588.9$ \\
Postoperative new onset dialysis & $125.5,389.5,392.7,394.2,399.5$ \\
Wound infection & $998.5,998.59$
\end{tabular}

ICD-9-CM, International Classific ation of Diseases, Ninth Revision, Clinical Modification; $C A B G$, coronary artery bypass graft; $A M I$, acute myocardial infarction; $C O P D$, chronic obstructive pulmonary disease; $P V D$, peripheral vascular disease; $C R F$, chronic renal failure; $A R F$, acute renal failure; $C P R$, cardiopulmonary resuscitation; $I A B P$, intra-aortic balloon pump; $L O S$, length of stay. ${ }^{*}$ Charlson Index includes a weighted compilation of 17 baseline comorbidities in multiple systems, including cardiovascular (ischemic disease and congestive heart failure), renal, endocrine (diabetes mellitus), cerebrovascular, peptic ulcer, rheumatologic, hepatic, oncologic (history of cancer or malignancies), and infectious (human immunodeficiency virus/acquired immune deficiency). A total of 1186 unique ICD-9 codes are used for the creation of the Charlson Index. 
TABLE E3. Variables included in propensity analysis to assess the likelihood of undergoing early coronary artery bypass grafting

Category Variable

Demographics

Age (y)

Gender

Race

Charlson index score*

Hypertension (not part of Charlson)

MI type

Anterolateral infarct

Subendocardial infarct

Acuity

Shock

Cardiac arrest on admission

Thrombolysis before CABG

Mechanical ventilation before CABG

Conduction disorder

Ventricular fibrillation on admission

Ventricular tachycardia on admission

Atrial fibrillation on admission

Angina on admission

Red blood cell transfusion before CABG

Angiogram before $\mathrm{CABG}$

Coronary stent before CABG

MI, Myocardial infarction; $C A B G$, coronary artery bypass grafting. *These variables include 17 Charlson index parameters to comprise the 35 variables used to create the propensity score. 